\title{
Efficiency of local surface plasmon polariton excitation on ridges
}

\author{
Radko, I.P.; Bozhevolnyi, S.I.; Brucoli, G.; Martin-Moreno, L.; Garcia-Vidal, F.J.; Boltasseva, Alexandra
}

Published in:

Physical Review B Condensed Matter

Link to article, DOI:

10.1103/PhysRevB.78.115115

Publication date:

2008

Document Version

Publisher's PDF, also known as Version of record

Link back to DTU Orbit

Citation (APA):

Radko, I. P., Bozhevolnyi, S. I., Brucoli, G., Martin-Moreno, L., Garcia-Vidal, F. J., \& Boltasseva, A. (2008).

Efficiency of local surface plasmon polariton excitation on ridges. Physical Review B Condensed Matter, 78(11), 115115. https://doi.org/10.1103/PhysRevB.78.115115

\section{General rights}

Copyright and moral rights for the publications made accessible in the public portal are retained by the authors and/or other copyright owners and it is a condition of accessing publications that users recognise and abide by the legal requirements associated with these rights.

- Users may download and print one copy of any publication from the public portal for the purpose of private study or research.

- You may not further distribute the material or use it for any profit-making activity or commercial gain

- You may freely distribute the URL identifying the publication in the public portal 


\title{
Efficiency of local surface plasmon polariton excitation on ridges
}

\author{
I. P. Radko, ${ }^{1, *}$ S. I. Bozhevolnyi, ${ }^{2, \dagger}$ G. Brucoli, ${ }^{3}$ L. Martín-Moreno, ${ }^{3}$ F. J. García-Vidal, ${ }^{4}$ and A. Boltasseva ${ }^{5}$ \\ ${ }^{1}$ Department of Physics and Nanotechnology, Aalborg University, Skjernvej 4A, DK-9220 Aalborg Øst, Denmark \\ ${ }^{2}$ Institute of Sensors, Signals and Electrotechnics, University of Southern Denmark, Niels Bohrs Allé 1, DK-5230 Odense M, Denmark \\ ${ }^{3}$ Departamento de Fisica de la Materia Condensada-ICMA, Universidad de Zaragoza, E-50009 Zaragoza, Spain \\ ${ }^{4}$ Departamento de Física Teórica de la Materia Condensada, Universidad Autónoma de Madrid, E-28049 Madrid, Spain \\ ${ }^{5}$ Department of Photonics Engineering, Technical University of Denmark, Building 345v, DK-2800 Kongens Lyngby, Denmark
}

(Received 3 June 2008; revised manuscript received 23 August 2008; published 19 September 2008)

\begin{abstract}
The issue of efficient local coupling of light into surface plasmon polariton (SPP) modes is an important concern in miniaturization of plasmonic components. Here we present experimental and numerical investigations of efficiency of local SPP excitation on gold ridges of rectangular profile positioned on a gold film. The excitation is accomplished by illuminating the metal surface normally with a focused laser beam. Wavelength dependence and dependence of the efficiency on geometrical parameters of ridges are examined. Using leakage radiation microscopy, the efficiency of $\sim 20 \%$ is demonstrated experimentally. Numerical simulations based on Green's tensor approach are in good agreement with the experiment and allow suggesting an optimization of parameters for improving the efficiency of SPP excitation.
\end{abstract}

DOI: 10.1103/PhysRevB.78.115115 PACS number(s): 42.82.Et, 73.20.Mf, 78.67.Bf, 42.25.Fx

\section{INTRODUCTION}

Plasmonics is a branch of nanophotonics that is concerned with manipulation of surface plasmon polaritons (SPPs) at nanoscale by means of specifically tailored metal structures. A great interest in this area is explained by two very interesting and promising applications for SPPs: integrated plasmonics and nanosensing. Integrated plasmonics, in contrast to integrated optics, operates with SPP waves, which can be squeezed much better than light, giving a possibility for high integration and in turn transmitting the signal much faster than electric current does. This provides a technology capable to replace electrical-circuit interconnections, which are strongly speed limited by the $R C$ delay, ${ }^{1}$ with plasmonic ones limited merely by the SPP frequency. ${ }^{2,3}$ Moreover, plasmonics can bridge microscale photonics and nanoscale electronics. ${ }^{2}$ Use of SPPs for nanosensing exploits surface plasmon resonances, which can be frequency shifted due to the presence of a substance being sensed as well as due to structure geometry. Furthermore, the latter, if chosen appropriately, can lead to an enormous field enhancement increasing otherwise very weak Raman signal. ${ }^{4}$ Both applications require high miniaturization of the components involved, and the issue of efficient local light coupling into SPP modes is an important concern.

A very well-known and widely used technique for excitation of SPPs is Kretschmann configuration. If a metal film illuminated from below is of appropriate thickness, and a parallel beam is used as a light source, almost all the incident power can be transmitted into plasmons, meaning that the efficiency of light to SPP coupling reaches nearly $100 \%$. However, it is not a local excitation since a parallel beam assumes its width to be at least a few hundreds of microns, whereas plasmonic applications are in lack of efficient compact sources of SPPs of the several-wavelength size. Moreover, the propagation length of a SPP beam along such a metal film is twice shorter than along an opaque one ${ }^{5}$ due to the additional radiation losses into the substrate, which in most cases is undesirable. Otto configuration, which in common with Kretschmann one uses a similar principle of evanescent coupling, bears the same disadvantage of nonlocal excitation.

A number of configurations have been developed for local light-SPP coupling. ${ }^{6}$ Among them are excitation using illumination through a probe of a scanning near-field optical microscope ${ }^{7}$ by a highly focused laser beam ${ }^{8,9}$ or by a radially polarized Bessel beam ${ }^{10}$ and excitation at discontinuities of a metal film, ${ }^{11}$ launching surface plasmons through an array of nanoholes, ${ }^{12}$ through a subwavelength slit supplemented with a periodic set of grooves ${ }^{13}$ or by a surface protrusion defect in form of a particle or a single ridge ${ }^{14}$ as well as by a periodic set of those. ${ }^{15}$ The efficiency of light-SPP coupling, defined as the ratio of SPP power to that of light, for some configurations has been evaluated both experimentally and numerically. ${ }^{9,10,15-17}$ The efficiency of the techniques that use radially polarized Bessel or highly focused parallel beams can be quite high. ${ }^{9,10}$ But apart from the already mentioned disadvantage of use of thin metal films, those methods, especially the former one, produce SPPs with virtually all directions of their $k$ vectors. This is very impractical for integrated plasmonics operating with SPP beamslaterally confined SPP waves with their $k$ vectors lying in a small range of directions (usually considerably below a radian)_but might be useful for nanosensing. In the work of Ditlbacher et al., ${ }^{15}$ the efficiency of SPP excitation mediated by gold ridges was studied. By illuminating the structures with a focused laser beam incident normally to the surface, two SPP beams propagating in opposite directions were excited. With only three ridges placed periodically on a gold film, the efficiency of light coupling into a single SPP beam was found to be $\sim 8 \%$. This is already a very good result if one bears in mind that it is a local coupling configuration, and such commercial devices in serial production could be virtually fabricated on a microchip. A very recent study of light coupling to SPP for a single subwavelength hole in a gold film ${ }^{16}$ revealed the efficiency of up to $28 \%$. Note, though, that this is the value normalized with respect to the 

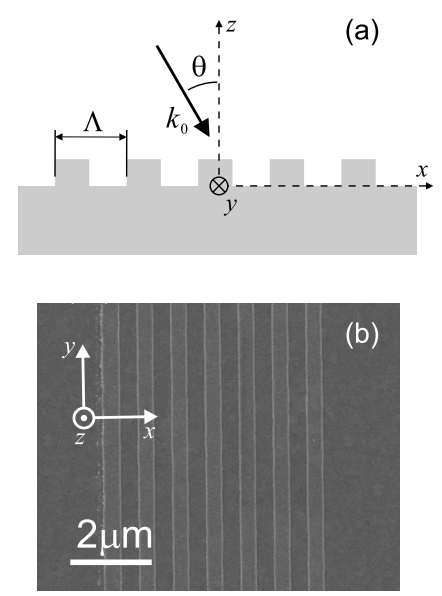

FIG. 1. (a) Geometry of the configuration under study and coordinate system. (b) Scanning electron microscope image of a typical structure under investigation. In this case it is a set of seven periodically arranged ridges. Period is $800 \mathrm{~nm}$.

power incident onto the hole area, meaning that the absolute efficiency (normalized with respect to the total incident power) is considerably smaller. In our further discussions we shall operate with absolute values of efficiency. Note that a possibility of grating geometry optimization has been investigated numerically. ${ }^{17}$ It was shown that a grating of indentations is more efficient than a grating of protrusions and an optimization of groove depth and width results in the coupling efficiency of $16 \%-22 \%$ to a single SPP beam.

This paper reports on the capability to optimize the ridge configuration for SPP excitation, found already promising, ${ }^{15}$ with respect to its efficiency. We chose our structures to have a form of protrusions rather than indentations because ridges are easier to fabricate using electron-beam lithography (EBL), a technique which is more available than focused ion beam required for grooves. We tried to optimize the geometrical parameters of an individual ridge (width and height), the number of those in a periodic array, as well as the working wavelength for a given array period. The results presented were obtained both numerically and experimentally.

\section{INVESTIGATION TECHNIQUES}

\section{A. Structures under investigation and experimental technique}

We consider single ridges and periodic sets of those on top of a gold film illuminated with a focused laser beam. In the case of multiple ridges, the interaction between light and SPPs is achieved by coupling through the grating ${ }^{5}$ and can be described approximately by the condition of momentum conservation,

$$
\vec{k}_{\mathrm{SPP}}^{\|}=\vec{k}_{0}^{\|}+\vec{u}_{x} \frac{2 \pi}{\Lambda} n,
$$

where $\vec{k}_{\text {SPP }}^{\|}$and $\vec{k}_{0}^{\|}$denote the wave-vector components in the $(x, y)$ plane [see Fig. 1(a)] of the scattered SPP and the incident light, respectively, $\Lambda$ is the grating period, and $n$ is an integer. Since the grating momentum has only $x$ component, we consider light incidence in the $(x, z)$ plane, hence $\vec{k}_{0}^{\|}$ $=\vec{u}_{x} k_{0} \sin \theta$ and Eq. (1) can be rewritten as

$$
k_{\mathrm{SPP}}=k_{0} \sin \theta+n G,
$$

with $G$ denoting the grating momentum $2 \pi / \Lambda$. As mentioned in Sec. I, excitation of laterally confined SPP waves is of most practical importance, therefore we need to focus the incident beam to a spot. On the other hand, we would like to have relatively small divergence of the excited SPP beam. Both conditions can be satisfied if we require that the SPP propagation length be of the order of the Rayleigh length of the SPP Gaussian beam, which gives the diameter of several microns for our wavelength range (see below). We use a 20 -fold objective [numerical aperture $(\mathrm{NA}=0.4)$ ], which fits well for these purposes. Due to the short working distance of the objective, the normal incidence of light is the only possibility for our configuration, thus from the Eq. (2) we obtain an approximate condition for the grating period, which should be a multiple of the SPP wavelength.

All structures were fabricated by EBL on a resist layer spun on top of a 50-nm-thick gold film on a quartz substrate, evaporation of a second gold layer, and subsequent liftoff. A scanning electron microscope image of a typical structure used in our investigations can be seen in Fig. 1(b).

The number of ridges to be used for SPP excitation is changing from 1 (single-ridge configuration) to at most 19. To find the exact optimum relation between the illumination wavelength and the grating period, it is enough to change only one of those parameters, and we choose to change the wavelength with the periodicity of ridges being fixed at $\Lambda$ $=800 \mathrm{~nm}$. Because of limitations of EBL the height of the structures cannot be changed within a sample, therefore in the experiment all ridges are $50 \mathrm{~nm}$ high, although, in numerical simulations it is a variable parameter.

To launch SPPs, we illuminate our structures with a tunable (wavelength range 700-860 nm) Ti:sapphire laser's Gaussian beam at normal incidence. Using the 20 -fold objective, the beam is focused to a spot with diameter estimated to be $\sim(5 \pm 0.5) \mu \mathrm{m}$ (at the level $1 / e^{2}$ of intensity). Since the structure under investigation is homogeneous along the $y$ direction, only $x$ component of the incident field will contribute into SPPs. Therefore the polarization of the incident laser beam is perpendicular to the ridges. In this case two SPP beams are excited propagating in opposite directions away from the structure. Note that in most application configurations only one of them can be used. For this reason we define the light-plasmon coupling efficiency as the ratio between the power carried by one of the SPP beams and that of the incident laser illumination, i.e., the percentage of power transferred from light into a SPP beam. In other words, we find a unidirectional SPP excitation efficiency. Note also that with extended structures (such as periodic set of ridges) the efficiency depends substantially on the position of the illumination spot. In this case we search for the position that gives the maximum efficiency. It is this unidirectional efficiency that we call the light-plasmon coupling efficiency or the SPP excitation efficiency.

To estimate the magnitude of the efficiency, we measure the power of the leakage radiation (LR) from the excited SPP 
beam into the glass substrate. The power of the LR is in strict relation with that of the SPP. ${ }^{18}$ So, if the metal film thickness and the SPP wavelength are known, it is easy to evaluate the power carried by a SPP beam from the measured power of the corresponding LR. The random error of all measurements discussed in this paper is estimated to be $\sim 15 \%$. A setup for making LR measurements is well known and described in details elsewhere. ${ }^{19}$

\section{B. Calculation technique}

All calculations are based on the two-dimensional (2D) electromagnetic Green's tensor (GT) approach. The general theory and the solution technique have been developed previously. ${ }^{20}$ The air-gold interface is set in the $(x, y)$ plane and the ridges are infinite along the $y$ axis, and thus all the properties are invariant in this direction. If the incident electric field propagates in the $(x, z)$ plane and has $p$ polarization [see Fig. 1(a)], then the total and the scattered fields will keep propagating in the $(x, z)$ plane with the same polarization. That allows us to assume that the system can be solved in a $2 \mathrm{D}$ subspace $\mathbf{r}=(x, 0, z)$ excluding the $y$ dependence in the calculations. For the incident electric field we assume (i) $E_{y}^{\text {in }}=0$ and (ii) $E_{x}^{\text {in }}(x, z)=e^{-\left(x-x_{0}\right)^{2} / \sigma^{2}} E_{x}^{\text {in }}(z)$; i.e., the field has Gaussian profile only in the $x$ direction. Even though $y$ components of the incident electric field in the experiment are nonzero with relatively weak focusing $(\sigma=2.5 \mu \mathrm{m})$, they are negligible. ${ }^{21}$ Since the thickness of the gold film in the experiment (of $50 \mathrm{~nm}$ ) is comparable to its skin depth, we approximate the gold slab with a semi-infinite gold half space. Let the dielectric constants of gold (as well as the gold ridges) and air be $\epsilon$ and 1 , respectively. When a system formed by two semi-infinite half spaces (air and gold) with embedded scatterers (ridges) at the interface is illuminated with an incident electric field $\mathbf{E}_{0}(\mathbf{r})$, the total field $\mathbf{E}(\mathbf{r})$ is given, according to Eq. (8) of Ref. 20, by the volumeintegral equation

$$
\mathbf{E}(\mathbf{r})=\mathbf{E}_{0}(\mathbf{r})+k_{0}^{2} \int_{A}(\epsilon-1) \mathbf{G}_{2 \mathrm{D}}\left(\mathbf{r}, \mathbf{r}^{\prime}\right) \mathbf{E}\left(\mathbf{r}^{\prime}\right) d \mathbf{r}^{\prime},
$$

where the integration runs only over the scatterers section $A$. $\mathbf{G}_{2 \mathrm{D}}\left(\mathbf{r}, \mathbf{r}^{\prime}\right)$ is the 2D GT that gives the field at a point $\mathbf{r}$ generated by an infinite line source extending in the $y$ direction centered at an arbitrary point $\mathbf{r}^{\prime}$. Equation (3) is a combination of the physical constraints governing our system that is the Maxwell equations and the boundary conditions.

The solution is found in a two-step process. (i) First, the electric field inside every ridge of the grating is computed by solving Eq. (3) for points $\mathbf{r}$ being placed inside the ridges. The calculation involves the GT connecting any two points inside the scatterers, which can be found (in direct space) in the integral representation ${ }^{22}$ and must be attained numerically. The integration technique has been described in details in Appendix B of Ref. 23. (ii) The field outside the ridges can then be found using the self-consistent field inside the ridges determined in the first step. Calculating the excitation efficiency only involves the analytical asymptotic approximation of the GT for the in-plane far-field zone, ${ }^{22,23}$ $\mathbf{G}_{2 \mathrm{D}}\left(x-x^{\prime} \gg \lambda, z \sim 0, z^{\prime} \sim 0\right) \rightarrow \mathbf{G}_{\mathrm{SPP}}$,

$$
\mathbf{E}_{\mathrm{SPP}}(\mathbf{r})=k_{0}^{2} \int_{A}(\epsilon-1) \mathbf{G}_{\mathrm{SPP}}\left(\mathbf{r}, \mathbf{r}^{\prime}\right) \mathbf{E}\left(\mathbf{r}^{\prime}\right) d \mathbf{r}^{\prime} .
$$

This GT describes the coupling between the field inside the ridges and the SPP field at remote points near the surface plane.

\section{EXPERIMENTAL AND NUMERICAL RESULTS}

\section{A. Wavelength dependence of coupling efficiency}

First, we investigated the wavelength dependence of the SPP excitation efficiency. For this purpose a 50-nm-high and 150-nm-wide single gold ridge was fabricated. A unidirectional SPP excitation efficiency was measured in the wavelength range 750-860 $\mathrm{nm}$ and found to decrease rapidly with the increasing wavelength. The most basic description of SPP launching in this configuration relies on a dipole scattering of light on the ridge. Its two sharp edges separated by $50 \mathrm{~nm}$ act as a dipole in the incident electric field oriented perpendicular to the ridge. Since the size of this dipole is considerably smaller than the wavelength, Rayleigh scattering gives an adequate description of the wavelength dependence of scattered light intensity. ${ }^{24}$ We plotted the experimentally obtained values along with the numerical data in a double-logarithmic chart to find the exponent in the powerlaw dependence [Fig. 2(a)]. Both plots are fitted with a straight line, which yields the magnitudes of the assumed exponents -5.4 and -4.5 for the experimental and numerical data, respectively. One can see that the agreement is fairly good both between each other and with Rayleigh scattering, which scales as a power of -4 of the wavelength. One should hardly expect the exact correspondence between the experimental and numerical data because the simulated results depend much on the dielectric constant of metal (which is known to depend on the fabrication) as well as on the exact profile of the ridge, which is not ideally rectangular in the experiment.

In order to increase the efficiency, it seems reasonable to use several ridges arranged periodically. As mentioned in Sec. II A, the period should be close to the SPP wavelength, but the precise value is not obvious. We investigated the wavelength dependence of light-plasmon coupling efficiency for structures composed of up to six ridges having the same geometrical parameters as in the previous experiment [Fig. 2(b)]. Having the feature of a single-ridge dependence (to decrease rapidly with wavelength), the efficiency is noticeably enhanced near the wavelength of $790 \mathrm{~nm}$ where the coherently excited (on different ridges) SPP waves interfere with each other constructively increasing the light-plasmon coupling. Note that while the number of ridges used is increasing, this effect starts to be more pronounced. For one ridge we obtained a unidirectional efficiency around $0.6 \%$ at the wavelength $800 \mathrm{~nm}$, which is almost four times less than that declared in Ref. 15. This is due to the difference in laser illumination spot size $(\sim 5 \mu \mathrm{m}$ in our case vs $1 \mu \mathrm{m}$ in Ref. 15). Although better focusing is preferred with a few ridges, a larger beam size might be more advantageous with the considerable number of ridges (10-20) since more scatterers are involved in the excitation process. 

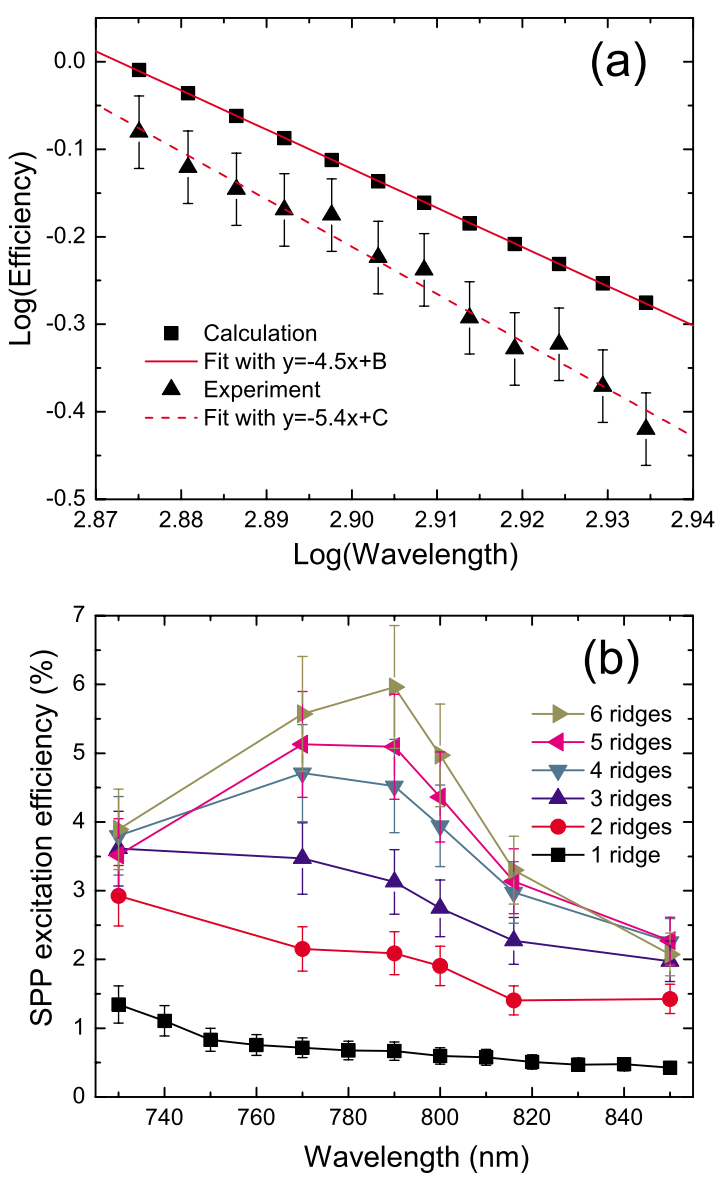

FIG. 2. (Color online) (a) Efficiency of SPP excitation on 50$\mathrm{nm}$-high and 150-nm-wide gold ridge versus the free-space wavelength used for excitation plotted in double-logarithmic scale along with a linear fit. (b) Efficiency of SPP excitation on sets of periodically arranged 50-nm-high 150-nm-wide gold ridges versus the free-space wavelength measured for the different number of ridges composing the grating. Separation between ridges is $\Lambda=800 \mathrm{~nm}$.

\section{B. Dependence on geometrical parameters of ridge}

Finding the optimum geometrical parameters of an individual ridge is important because, as we show in this section, geometrical optimization can sufficiently increase the efficiency of light-plasmon coupling. We performed numerical simulations of SPP excitation on a single ridge whose height is varying in the range $50-230 \mathrm{~nm}$ and width, in the range of 200-600 nm, and calculated its efficiency [Fig. 3(a)]. With only small difference, ridges of all heights feature maximum efficiency when the width is close to $350 \mathrm{~nm}$, i.e., almost half of the wavelength. The maximum achievable efficiency for every given height is then plotted in Fig. 3(b). One can see almost a sixfold increase in the efficiency with the height growing from 50 up to $130 \mathrm{~nm}$, where it reaches its maximum.

We checked the width dependence of excitation efficiency experimentally also using illumination at the wavelength 800 $\mathrm{nm}$. The ridge height was fixed at $50 \mathrm{~nm}$. We tested configurations with one, three, and five ridges aligned periodically (period $\Lambda=800 \mathrm{~nm}$ ) in the two latter cases [Fig. 3(c)]. The experimental data exhibit maximum efficiency for the ridge
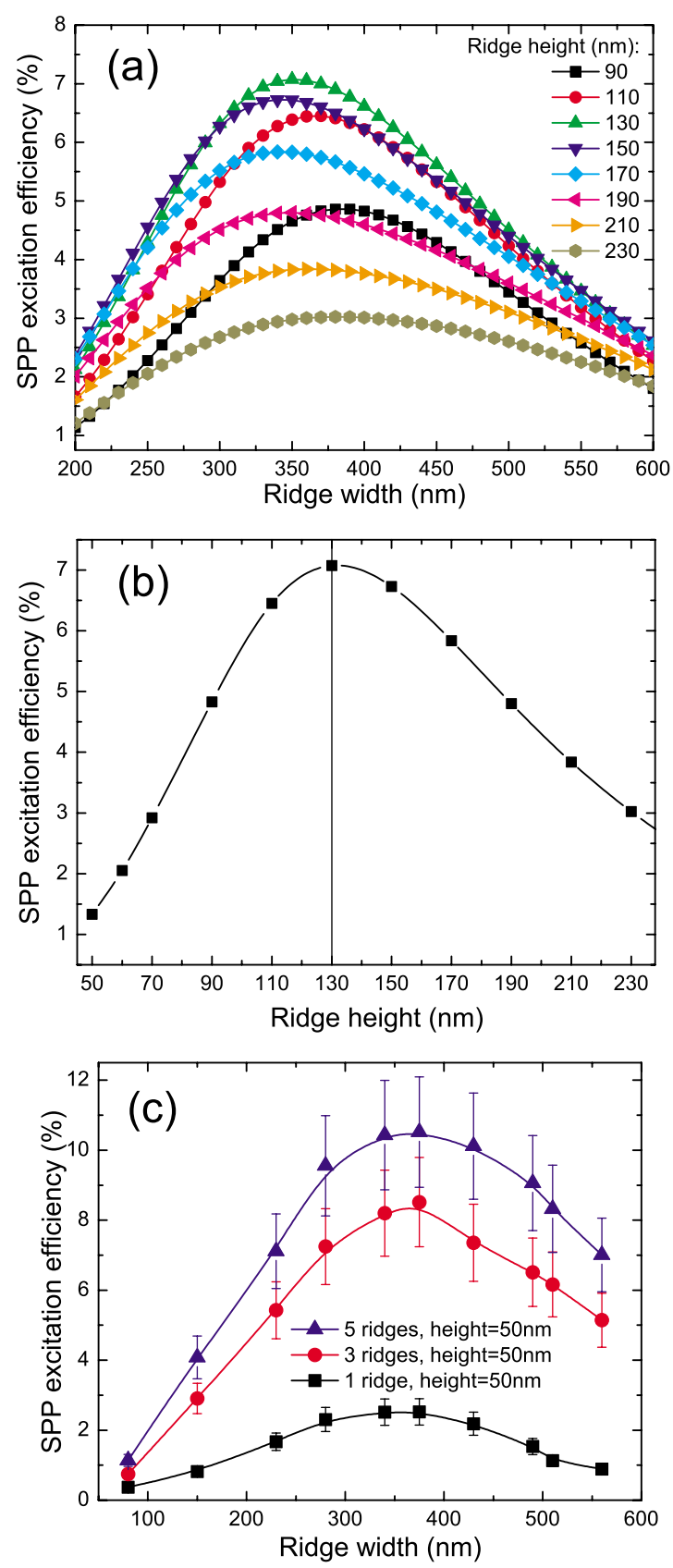

FIG. 3. (Color online) (a) Calculation of efficiency of SPP excitation on a single ridge versus its width. Different curves show data for different ridge heights. (b) Calculation of maximum attainable efficiency (with all possible ridge widths) of the SPP excitation on a single ridge versus its height. (c) Experimental results for dependencies of SPP excitation efficiency on the ridge width. The ridge height is fixed at $50 \mathrm{~nm}$. Three curves show three sets of measurements accomplished on a single ridge and on three and five ridges aligned in gratings with the period $\Lambda=800 \mathrm{~nm}$. The freespace wavelength is $800 \mathrm{~nm}$ both in calculations and in the experiment.

width close to $350 \mathrm{~nm}$, which is in agreement with the numerical simulations. The figure also demonstrates that the optimum width is not changing for the increasing number of ridges at least within a small quantity. Note that the fact that we obtained larger efficiency experimentally, than it is pre- 

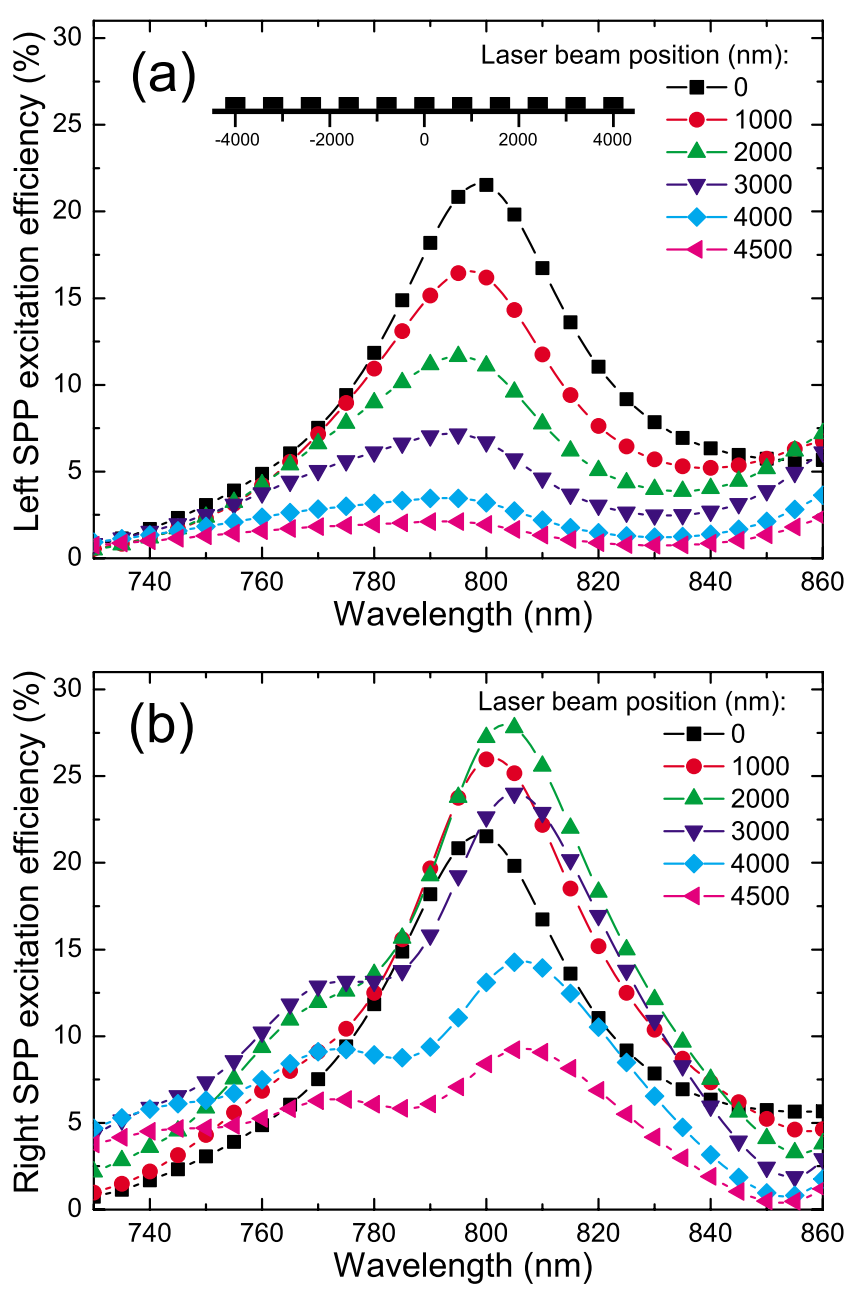

FIG. 4. (Color online) Numerical results for wavelength dependence of efficiency of SPP excitation on 11 ridges (height $=50 \mathrm{~nm}$ and width $=280 \mathrm{~nm}$ ). Different positions of a laser beam (diameter $=5 \mu \mathrm{m}$ ) illuminating the sample are shown by different curves. Calculations accomplished for SPP propagating (a) to the left and (b) to the right from the grating. Inset of the panel (a) shows the geometry and coordinate system.

dicted numerically for a 50-nm-high ridge, can be explained by a slightly smaller laser-beam diameter used in the experiment.

\section{Optimum wavelength for excitation on ridges}

As we already mentioned, one of the ways to increase the efficiency of excitation is to use a periodic set of ridges. However, the optimum period is not necessarily equal to the SPP (or free-space) wavelength. To get more insight into that, we investigate arrays of ridges with the fixed period of $\Lambda=800 \mathrm{~nm}$ and change the laser-beam wavelength instead since array period cannot be changed in the experiment.

First, we investigated numerically SPP excitation on a grating composed of 11 ridges having width of $280 \mathrm{~nm}$ and height of $50 \mathrm{~nm}$. The diameter of the modeled laser beam is $5 \mu \mathrm{m}$. We placed the zero of the coordinate system to the center of the grating [inset of Fig. 4(a)]. Then we performed a scan of the laser beam from the center toward increasing coordinates evaluating the powers carried by the two SPP beams propagating in opposite directions for different wavelengths [Figs. 4(a) and 4(b)]. Expectedly, the efficiency drops down monotonously for the left-propagating SPP beam [see the inset of Fig. 4(a) for the geometry] with the laser beam moving from the center of the grating to the right [Fig. 4(a)]. On the contrary, the efficiency for the right-propagating SPP increases first, reaching the maximum at the coordinate $\sim 2000 \mathrm{~nm}$, and then monotonously decreases [Fig. 4(b)]. We will get back to this behavior in Sec. III D, whereas now a more important result is the wavelength where the maximum efficiency is observed: it is $805 \mathrm{~nm}$. Note that in this optimum case, the grating period of $800 \mathrm{~nm}$ is between the free-space wavelength $805 \mathrm{~nm}$ and the corresponding SPP wavelength $\sim 789 \mathrm{~nm}$.

In the experiment, we investigated two gratings composed of 7 and 11 ridges of the width $200 \mathrm{~nm}$ and $280 \mathrm{~nm}$ each, respectively. Instead of scanning the laser beam across the gratings, we were searching the position with the maximum achievable efficiency and then picked that efficiency value to the chart. Thus, the way it was measured gives an envelope of all the curves in Fig. 4(b). The result obtained is shown in Fig. 5(a) together with the envelope for comparison. The lower curve yields the optimum wavelength of $790 \mathrm{~nm}$, which repeats the result shown in Fig. 2(b). The experimental curve for the 11-ridge gratings shows that the optimum wavelength shifts toward higher values with the increasing number of ridges. Comparison with the envelope of the curves in Fig. 4(b) (obtained for the same geometry, i.e., 11 ridges of the width $280 \mathrm{~nm}$ and height $50 \mathrm{~nm}$ ) shows good agreement in both the optimum wavelength and the general behavior of the spectrum. For lower wavelengths the experiment gives higher efficiencies. This is because the experimental structure always has small irregularities in the ridge periodicity, which makes the spectrum broader and, as a result, its wings higher.

A drift of the optimum wavelength with the increasing number of ridges can be also observed in another series of measurements where we investigated a dependence of lightSPP coupling efficiency on the number of ridges involved in the process [Fig. 5(b)]. The ridges participating in the excitation are $280 \mathrm{~nm}$ wide. First of all, at wavelengths far from the optimum one, the efficiency comes very quickly to saturation $(730$ and $770 \mathrm{~nm}$ ) or even drops down $(850 \mathrm{~nm})$ with the number of ridges exceeding five. Second, for the small number of ridges (below seven) the optimum wavelength is somewhere around $800 \mathrm{~nm}$ but cannot be clearly defined: the gratings are broadband. However, with the increasing number of ridges, the wavelength dependence gets more pronounced: first, $800 \mathrm{~nm}$ is preferable (for 11 ridges) and then $810 \mathrm{~nm}$ (for 15 and more ridges). The two main conclusions are: (i) the optimum wavelength (or the optimum grating period for a given wavelength) is growing (diminishing) with the increasing number of ridges composing the grating and (ii) for a grating with large number of ridges the SPP wavelength corresponding to the optimum illumination is approaching the grating periodicity from the low-wavelength side. The latter means that there are two competing processes of electromagnetic interaction between ridges: one is on the dielectric (air) side and mediated by photons, and the other is 

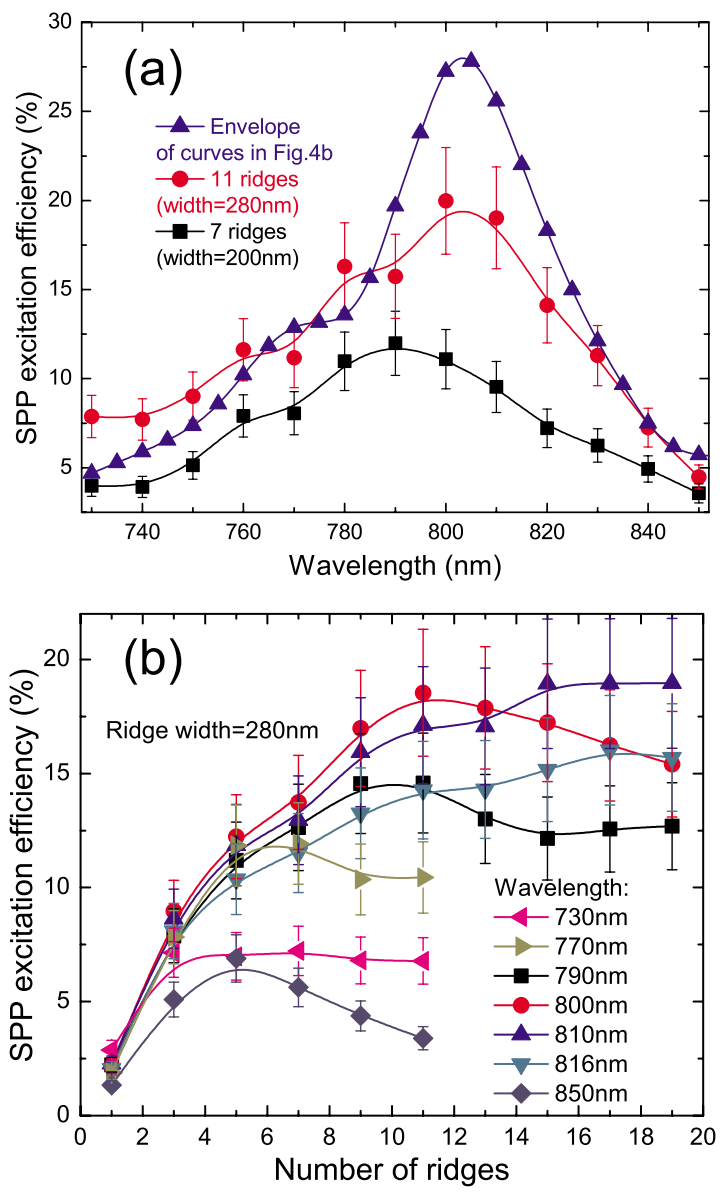

FIG. 5. (Color online) (a) Experimental results for wavelength dependence of efficiency of SPP excitation on two different gratings $(\Lambda=800 \mathrm{~nm})$ composed of 7 (height $=50 \mathrm{~nm}$ and width=200 nm) and 11 ridges (height $=50 \mathrm{~nm}$ and width $=280 \mathrm{~nm}$ ) plotted together with the envelope of curves shown in Fig. 4(b) for comparison. (b) Experimental results for efficiency of SPP excitation versus the number of ridges in the grating $(\Lambda=800 \mathrm{~nm})$ used for excitation. Ridge with height $=50 \mathrm{~nm}$ and width $=280 \mathrm{~nm}$. Results obtained at different wavelengths are shown by different curves.

on the metal side occurring through SPPs. To enhance each of those processes, the grating period equal to the corresponding wavelength is required, which sets the optimum period in between of them. As it turns out, however, the interaction through plasmons is stronger, and with the large number of ridges it takes over the process equating the optimum period with the SPP wavelength. One of the reasons that we obtained the maximum efficiency at $\lambda_{0}=810 \mathrm{~nm}$ instead of $816 \mathrm{~nm}$ (corresponding to $\lambda_{\mathrm{SPP}}=\Lambda=800 \mathrm{~nm}$ ) might be a small misalignment (of about $0.5^{\circ}$ ) of the objective focusing illumination onto the grating [see Eq. (2)].

\section{Directionality of SPP excitation on periodic sets of ridges}

We conclude our consideration of efficiency of light-SPP coupling on ridges by demonstrating the extent of asymmetry of surface plasmon beams excitation while using such structures. This gives an idea of the total (bidirectional) lightplasmon coupling efficiency and also demonstrates the sen-
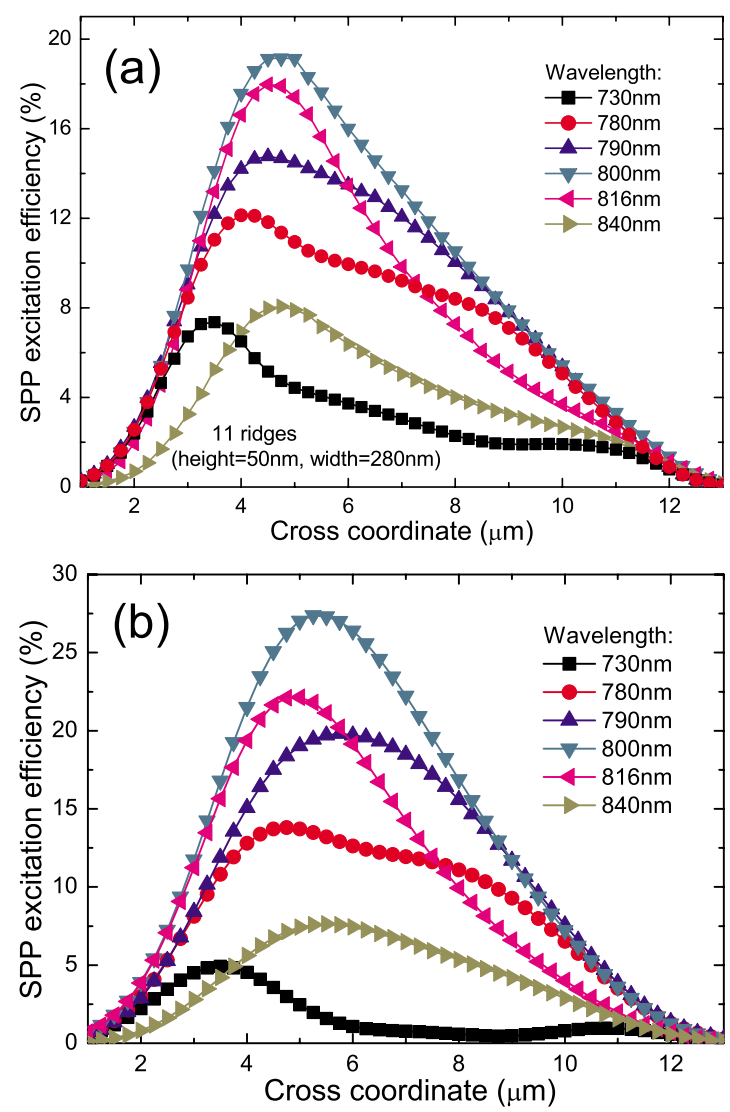

FIG. 6. (Color online) (a) Experimental and (b) numerical results for SPP excitation efficiency versus the position of a laser beam scanned across the grating $(\Lambda=800 \mathrm{~nm})$ composed of 11 ridges (height $=50 \mathrm{~nm}$ and width $=280 \mathrm{~nm}$ ). In the experiment the grating extends approximately from 3 to $11 \mu \mathrm{m}$ of the cross coordinate; in the numerical simulations this is the exact position of the grating. The error bars in (a) are omitted to not obscure the image.

sitivity of efficiency to the position of the laser beam with respect to the illuminated structure

For this purpose, we measure the efficiency while the laser beam is scanned across a grating [Fig. 6(a)]. Such profiles were recorded at several wavelengths. The grating was composed of 11 ridges (height $=50 \mathrm{~nm}$ and width $=280 \mathrm{~nm}$ ). At two wavelengths - at which the highest efficiencies were obtained (800 and $816 \mathrm{~nm}$ ) - the profiles feature a relatively sharp maximum. At the optimum wavelength the efficiency is very sensitive to the laser-beam position, which leads to a more unidirectional excitation. Assuming that the center of the grating is approximately positioned at $x=7 \mu \mathrm{m}$, one can find the efficiency of SPP excitation on the farthest end of the grating while having a maximum at the nearest one: $7 \%$ versus $19 \%$ at $\lambda_{0}=800 \mathrm{~nm}$. For the wavelengths out of the resonance, the profiles possess a small plateau in the middle forming a shape reminding trapezium. At the wavelength 730 nm one can even notice a very small second local maximum on the other end of the grating.

Modeling of this geometry shows an excellent agreement [Fig. 6(b)] in curves' shape including the two local maxima at $\lambda_{0}=730 \mathrm{~nm}$. The explanation for the latter could be that out of the resonance the excitation on a fewer number of 
ridges is more efficient than on the whole grating because the periodic set of ridges causes destructive interference. Therefore, moving the beam out of the grating excludes most of the ridges from the process, which turns out to be more favorable in this case.

\section{CONCLUSIONS}

Summarizing, we have investigated numerically and experimentally the efficiency of SPP excitation on single ridges and on periodic sets of those. The variable parameters were wavelength of light used for excitation, ridges width and height, as well as the number of them in periodic arrangements. The maximum experimental unidirectional SPP excitation efficiency of about $20 \%$ was obtained for 11 ridges (width=280 $\mathrm{nm}$ and height=50 nm). Although the dependence of efficiency on ridge height was not investigated experimentally, numerical simulations show that it is an important parameter, which can lead to almost sixfold increase in efficiency for one ridge when changing the height from 50 to $130 \mathrm{~nm}$. The optimum ridge width was found to be close to half the illumination wavelength but somewhat smaller. This is in agreement with the result obtained previously for another working wavelength. ${ }^{17}$ For periodic sets of ridges used for SPP excitation, the SPP wavelength corresponding to the optimum free-space wavelength was found to be smaller than the ridge periodicity but approaching that with the increasing number of ridges.
It is difficult to extend the conclusions to systems distinct from the suggested one without thorough investigations. One can, however, conclude that efficient local excitation of SPPs is achievable with a moderately focused laser beam and only a few ridges. Regarding optimum parameters, it is clear that with the oblique incidence of the laser beam, the grating period and/or the excitation wavelength has to be adjusted according to Eq. (2). It is further expected that the ridge parameters (height and width) are mainly influenced by the choice of wavelength (most probably scaling with it linearly), but the precise values have to be found with detailed simulations.

Finally, we note that the value of efficiency of $20 \%$ found experimentally might be improved in the case of opaque metal film, ${ }^{17}$ which prevents the excitation of SPPs on a metal-substrate interface. The higher value of $\sim 28 \%$ obtained numerically for the same configuration is a good evidence of that. However, the use of opaque metal films will make the LR microscopy inapplicable for experimental investigations.

\section{ACKNOWLEDGMENTS}

This work was supported by the Danish Technical Research Council (Contract No. 26-04-0158), the European Network of Excellence, Plasmo-Nano-Devices (Contract No. FP6-2002-IST-1-507879), the Spanish MCyT under Projects No. MAT2005-06608-C02 and No. AP2005-55-185, and the NABIIT project (Contract No. 2106-05-033 from the Danish Research Agency).

\footnotetext{
*ilya@nano.aau.dk

${ }^{\dagger}$ Also at Department of Physics and Nanotechnology, Aalborg University, Skjernvej 4A, DK-9220 Aalborg Øst, Denmark.

${ }^{1}$ R. W. Keyes, Proc. IEEE 89, 227 (2001).

${ }^{2}$ R. Zia, J. A. Schuller, A. Chandran, and M. L. Brongersma, Mater. Today 9, 20 (2006).

${ }^{3}$ W. L. Barnes, A. Dereux, and T. W. Ebbesen, Nature (London) 424, 824 (2003).

${ }^{4}$ S. Lal, S. Link, and N. J. Halas, Nat. Photonics 1, 641 (2007).

${ }^{5}$ H. Raether, Surface Plasmons on Smooth and Rough Surfaces and on Gratings (Springer-Verlag, Berlin, 1988).

${ }^{6}$ A. V. Zayats and I. I. Smolyaninov, J. Opt. A, Pure Appl. Opt. 5, S16 (2003).

${ }^{7}$ B. Hecht, H. Bielefeldt, L. Novotny, Y. Inouye, and D. W. Pohl, Phys. Rev. Lett. 77, 1889 (1996).

${ }^{8}$ H. Kano, S. Mizuguchi, and S. Kawata, J. Opt. Soc. Am. B 15, 1381 (1998)

${ }^{9}$ H. Kano, D. Nomura, and H. Shibuya, Appl. Opt. 43, 2409 (2004).

${ }^{10}$ Q. Zhan, Opt. Lett. 31, 1726 (2006).

${ }^{11}$ L. Salomon, G. Bassou, H. Aourag, J. P. Dufour, F. de Fornel, F. Carcenac, and A. V. Zayats, Phys. Rev. B 65, 125409 (2002).

${ }^{12}$ E. Devaux, T. W. Ebbesen, J.-C. Weeber, and A. Dereux, Appl. Phys. Lett. 83, 4936 (2003).
}

${ }^{13}$ F. López-Tejeira et al., New J. Phys. 10, 033035 (2008).

${ }^{14}$ H. Ditlbacher, J. R. Krenn, N. Felidj, B. Lamprecht, G. Schider, M. Salerno, A. Leitner, and F. R. Aussenegg, Appl. Phys. Lett. 80, 404 (2002).

${ }^{15}$ H. Ditlbacher, J. R. Krenn, A. Hohenau, A. Leitner, and F. R. Aussenegg, Appl. Phys. Lett. 83, 3665 (2003).

${ }^{16}$ A.-L. Baudrion, F. de León-Pérez, O. Mahboub, A. Hohenau, H. Ditlbacher, F. J. García-Vidal, J. Dintinger, T. W. Ebbesen, L. Martín-Moreno, and J. R. Krenn, Opt. Express 16, 3420 (2008).

${ }^{17}$ G. Lévêque and O. J. F. Martin, J. Appl. Phys. 100, 124301 (2006).

${ }^{18}$ E. Kretschmann, Z. Phys. 241, 313 (1971).

${ }^{19}$ A. Drezet, A. Hohenau, A. L. Stepanov, H. Ditlbacher, B. Steinberger, N. Galler, F. R. Aussenegg, A. Leitner, and J. R. Krenn, Appl. Phys. Lett. 89, 091117 (2006).

${ }^{20}$ M. Paulus and O. J. F. Martin, Phys. Rev. E 63, 066615 (2001).

${ }^{21}$ M. Mansuripur, J. Opt. Soc. Am. A 3, 2086 (1986).

${ }^{22}$ T. Søndergaard and S. I. Bozhevolnyi, Phys. Rev. B 69, 045422 (2004).

${ }^{23}$ A. Y. Nikitin, G. Brucoli, F. J. García-Vidal, and L. MartínMoreno, Phys. Rev. B 77, 195441 (2008).

${ }^{24}$ J. D. Jackson, Classical Electrodynamics, 3rd ed. (Wiley, New York, 1998). 